Pure Sciences

Poster

Abstract ID: 114

\title{
Biotechnological potential of Kuantan mangrove actinomycete, Micromonospora K3-13
}

Suhaila Mohd Omar | Zaima Azira Zainal Abidin | Nur Fathiah Abdul Malek | Norsyafawati Mohd Farouk | Zarina Zainuddin I Ahmed Jalal Khan Chowdhury

Department of Biotechnology, Kulliyyah of Science, International Islamic University Malaysia

Introduction: Mangrove is a complex and unique ecosystem which experiences periodical tidal flooding inundated with low, moderate and high salinity water. Its muddy soil is rich in organic matter derived from the efficient nutrient cycling system and decomposition of mangrove leaves. An adaptation of mangrove microorganisms towards these environmental factors could promote the production of interesting bioactive compounds and enzymes. Methods: This study determines to unravel the identity and biotechnological potential of a Kuantan mangrove actinomycete, strain K3-13, through morphology observation, molecular identification, antimicrobial test and xylan degradation test. Results: The orange-colored mycelium-forming isolates produced spores after a long incubation period, and showed characteristics that resembled Micromonaspora colonies. Phylogenetic analysis of the 16S rRNA gene sequence of strain K3-13 indicated that the highest similarity was to Micromonospora carbonacea DSM43168 (99\%). It was observed that K3-13 produced blue diffusible pigment upon cultivation on agar media. Cross streak assay conducted to detect antimicrobial activity indicated that $\mathrm{K} 1-13$ has strong antibacterial activity against Bacillus subtilis and Staphylococcus aureus. K3-13 also showed positive xylanase activity by exhibiting decolourization zone in the agar-based assay using azo-xylan as substrate. Conclusions: On the basis of its ability to produce blue pigment, antimicrobial activity against at least two test organisms and positive xylan degrading activity, Micromonaspora K3-13 is concluded as strain worth exploring for its potential biotechnological application as a natural dye, antibacterial drugs and Xylan biodegraders.

KEYWORDS: Actinomycete, Mangrove, Micromonospora, biotechnological application 